\title{
ON THE SOLVABILITY OF SOME PARTIAL DIFFERENTIAL INEQUALITY
}

\author{
Martin HimmeL* \\ FB 08 - Institut für Mathematik, Johannes Gutenberg-Universität Mainz, \\ Staudinger Weg 9, D-55099 Mainz, Germany \\ * corresponding author: himmel@mathematik.uni-mainz.de
}

ABstract. The Dulac criterion is a classical method for ruling out the existence of periodic solutions in planar differential equations. In this paper the applicability and therefore reversibility of this criterion is under consideration.

KEYWORDs: dynamical systems, planar differential equations, limit cycles, Dulac's inequality, locally sufficient criteria.

\section{Introduction and Motivation}

Let $X: \mathcal{D} \rightarrow \mathbb{R}^{2}$ be a smooth vector-valued function with components $P$ and $Q, X=(P, Q)$, defined on some planar domain $\mathcal{D} \subseteq \mathbb{R}^{2}$. Now, consider the system of two ordinary differential equations

$$
\frac{d x}{d t}=P(x, y), \quad \frac{d y}{d t}=Q(x, y) .
$$

Such systems appear frequently in applications, e.g. in electrical engineering, physics, biology and many others, but they have also become a field of mathematical interest on their own. For simplicity, one can think of $P$ and $Q$ either just as polynomials of two real variables $x$ and $y$, or as smooth or even analytical functions given by some power series that converges in $\mathcal{D}$, i.e., $X$ is of class $\mathcal{C}^{r}$ with $r \in\{1,2, \ldots \infty, \omega\}$. Geometrically speaking, the solutions of the system (1) are smooth curves in the plane that are tangential to the function $X$ at each point $z=(x, y) \in \mathcal{D}$. If the components of $X$ are polynomials in $x$ and $y$ of degree one then

$$
X(x, y)=\left(\begin{array}{l}
a x+b y \\
c x+d y
\end{array}\right)
$$

with $a, b, c, d \in \mathbb{R}$ being real constants, and the analysis of system (1) is easy and the solution curves of system (1) are given explicitly in terms of the matrix exponential function $\mathrm{e}^{A}:=\sum_{k=0}^{\infty} \frac{1}{k !} A^{k}$. The situation changes drastically if $X$ is at least quadratic, i.e., if $P$ and/or $Q$ are polynomials of degree two or higher, $P, Q \in \mathbb{R}_{n}[x, y], n \geq 2$. Many things are known about quadratic differential equations, see [1] 3 , but they are still a broad area of research. Quite a few people have dedicated their whole life to the investigation of quadratic planar differential equations. Interest in this field is related to the 16th Hilbert problem, which is still unsolved even in the quadratic case.

\subsection{Sixteenth Hilbert problem}

Let $R_{d}[x, y]$ denote the space given by polynomials in $x$ and $y$ of degree at most $d$, and consider a planar sys- tem (1) with polynomial right-hand side $X \in \mathbb{R}_{d}^{2}[x, y]$. The 16th Hilbert problem consists of two parts, the first of which is a purely algebraic problem and deals with questions related to the topology of algebraic curves and surfaces. In this paper, we are mainly concerned about the second part of the 16th Hilbert problem, which deals with the curves that arise as solutions of the planar differential equation (1) with polynomial vector field $X$. More precisely, in the second part of his problem Hilbert asks for the maximal number and relative position of the isolated closed orbits, called limit cycles $1{ }^{1}$ this differential system (1) can have at most. It is even difficult to understand why fixed planar differential equations with polynomial vector field can only have a finite number of limit cycles. A proof of this fact is due to Ilyashenko in 1991 4], who actually corrected a very complicated and long proof due to Dulac [5], a student of Poincaré. Écalle et al. independently obtained a proof for this theorem [6]. Note that this does not imply the existence of an upper bound for the maximal number of limit cycles $H(d)$ which a planar polynomial system of degree $d$ can have. One calls $H(d)$ the $d$-th Hilbert number. Linear vector fields have no limit cycles; hence $H(1)=0$. Quadratic systems can actually have four limit cycles and some people believe that this is the maximal number of limit cycles a quadratic system can have, but it is still unknown whether or not $H(2)$ is a finite number. Usually, the first part of the 16th Hilbert problem is studied by researchers in real algebraic geometry, while the second part is considered by mathematicians working in dynamical systems or differential equations. Hilbert also pointed out that there exist possibly connections between these two parts. See paper [7] and [8] for the original paper in Russian by Ilyashenko and more recently a paper [9] for a survey about the second part of the 16th Hilbert

\footnotetext{
${ }^{1}$ In the past, the term limit cycle was used for a stable isolated closed orbit or just for a closed orbit. Nowadays and in this paper, a limit cycle is a closed orbit that is isolated in the set of all periodic orbits of some differential equation.
} 
problem.

\subsection{DULAC CRITERION}

In 2008, the author was confronted with the analysis of a quadratic polynomial differential equation. In literature there existed a quite technical proof showing the uniqueness of a limit cycle for this system. Then, fortunately, he was able to obtain the same result by applying the well-known

Theorem 1 (Dulac criterion [5]). Let $\Omega \subseteq \mathbb{R}^{2}$ be a simply connected region, $X:=(P, Q) \in \mathcal{C}^{1}\left(\Omega, \mathbb{R}^{2}\right)$ a smooth vector field and $B \in \mathcal{C}^{1}(\Omega, \mathbb{R})$ a smooth real-valued function - such that the partial differential inequality

$$
\operatorname{div}(B X):=\frac{\partial(B P)}{\partial x}+\frac{\partial(B Q)}{\partial y}>0
$$

is satisfied in $\Omega$. Then the planar ordinary differential equation (1) with $X$ as right-hand-side does not posses any periodic solution that is fully contained in $\Omega$.

Remark 1. (1.) The proof of theorem 1 is indirect: One assumes the existence of a closed orbit of (1) in $\Omega$ and applies the divergence theorem of Gauß. This immediately gives a contradiction.

(2.) In 2009, the author was able to weaken the assumptions made on the function $B$, see 10 . Essentially, the statement of theorem 1 still holds if the function $B$ is only weakly differentiable of first degree and equation (2) holds only almost everywhere in $\Omega$, compare definition 1

(3.) If the domain $\Omega \subseteq \mathbb{R}^{2}$ referred to in theorem 1 is not simply connected, but $p$-connected for some $p \in \mathbb{N}, p \geq 2$, then there can be at most $p-1$ closed orbits fully contained in $\Omega$.

(4.) The Dulac criterion is a generalization of the Bendixson criterion [11. In fact, the Bendixson criterion follows from theorem 1 if we choose $B=\mathbb{1}$, the function that is constant 1 for all $z \in \Omega$.

Definition 1 (Dulac function). The multiplier $B \in W^{1, p}(\Omega, \mathbb{R})$ is called Dulac function of the planar dynamical system $(1)$ in $\Omega \subseteq \mathcal{D}$ if and only if there is a real-valued continuous function $g>0$ having a positive sign in $\Omega$ except on a set of Lebesgue measure zero such that the equation

$$
\operatorname{div}(B X)=B \cdot \operatorname{div} X+\langle\nabla B, X\rangle=g
$$

holds in $\Omega$. Here $W^{1, p}(\Omega, \mathbb{R})$ denotes the Sobolev space of $L^{p}$ functions that are weakly differentiable of first degree and with a weak derivative in $L^{p}$. Vice versa, we call any function $B$ of class $W^{1, p}$ satisfying (3) a $g$-Dulac function of $X$ in $\Omega$.

In general, it is very difficult to find a Dulac function for some given vector field $X$, but if one incidentally guesses such a function, it is quite easy to verify that it obeys condition (2) ${ }^{2}$ Just for curiosity, the author wondered whether the converse statement of theorem 1 is also true.

Question 1. Given a smooth planar vector field $X$ and assume that the planar differential equation (1) does not have any periodic solution in some simply connected domain $\Omega$. Does there exist a Dulac function in $\Omega$ ?

The answer is No, if the boundary of $\Omega$ is formed by a periodic solution of the corresponding system, but in every other case considered by the author he was able to obtain an affirmative answer to question 1 raised above. In the following we quote some of these positive results.

\section{LOCAL RESULTS}

Gradient systems never possess periodic solutions [12], hence we expect that gradient fields have a Dulac function. Indeed, this is the case.

Theorem 2 (Gradient fields 10 ). Let $X=\nabla V$ be a globally defined gradient field with potential $V \in$ $\mathcal{C}^{2}\left(\mathbb{R}^{2}, \mathbb{R}\right)$. Then $B_{1}=\exp (V), B_{2}=\exp (-V)$ and $B_{3}=V$ are local Dulac functions for $X$ in $\Omega_{i} \subset \mathbb{R}^{2}$, $i=1,2,3$, with $\cup_{i=1}^{3} \Omega_{i}=\mathbb{R}^{2}$.

Proof. Define $B_{1}=\exp (V), B_{2}=\exp (-V)$ and $B_{3}=V$ and set $\Omega_{i}:=\left\{z \in \mathbb{R}^{2} \mid \operatorname{div}\left(B_{i} X\right)(z)>0\right\}$. Verify that $\cup_{i=1}^{3} \Omega_{i}=\mathbb{R}^{2}$ gives indeed the whole plane.

A standard result from calculus says that vector fields can be straightened locally unless there is an equilibrium. This fact can be used to obtain a local existence result in domains not containing zeros of the vector field. Such domains are called canonical regions.

Theorem 3 (Parallel flow [13]). Let $X: \mathcal{D} \subseteq$ $\mathbb{R}^{2} \rightarrow \mathbb{R}^{2}$ be any smooth vector field and $\Omega$ a canonical region of $X$, i.e., a region where the flow of system (1) is equivalent to a parallel flow in the sense of Neumann [14]. Then, under some integrability assumptions, $X$ has a Dulac function in $\Omega$.

Proof. By assumption there are no equilibria in the domain, hence the vector field can be straightened locally. Observe that, by this process of straightening, the planar system (1) decouples and the partial differential equation (3) from the definition of a Dulac function reduces to the linear ordinary differential equation

$$
B \operatorname{div} X+\alpha B_{r}=g
$$

\footnotetext{
${ }^{2}$ One can compare this problem to the decomposition of a natural number into its prime factors: If some natural number $n$ together with some product $p:=\prod_{i=1}^{N} p_{i}^{d_{i}}$ of powers of prime numbers is given, it is quite easy to decide whether $p$ is the prime factorization of $n, p=n$, but, from our current state of knowledge, it is very difficult to decompose a big natural number into its prime factors.
} 


$$
\begin{gathered}
b_{20}=\frac{a^{4}+4 a^{3} d-a^{2}\left(2 b c-c^{2}-3 d^{2}\right)+3 a c d(c-b)+c^{2}\left(b^{2}-b c+d^{2}\right)}{(a+d)\left(3 a^{2}+10 a d-4 b c+3 d^{2}\right)} \\
b_{02}=\frac{a^{2}\left(b^{2}+3 d^{2}\right)+a d\left(3 b^{2}-3 b c+4 d^{2}\right)-b^{3} c+b^{2}\left(c^{2}+d^{2}\right)-2 b c d^{2}+d^{4}}{(a+d)\left(3 a^{2}+10 a d-4 b c+3 d^{2}\right)} \\
b_{11}=\frac{2 a^{3} b+a^{2} d(7 b+3 c)-a\left(3 b^{2} c+b\left(c^{-} 3 d^{2}\right)-7 c d^{2}\right)-c d\left(b^{2}+3 b c-2 d^{2}\right)}{(a+d)\left(3 a^{2}+10 a d-4 b c+3 d^{2}\right)} \\
b_{01}=b_{10}=b_{00}=0 .
\end{gathered}
$$

Figure 1. Values of $b_{i j}$ from Equation (6).

which implies

$$
B_{r}=\frac{g-B \operatorname{div} X}{\alpha} .
$$

This is an ordinary differential equation with the solution

$$
B=\mathrm{e}^{a(r)}\left\{\int g \cdot \mathrm{e}^{a(r)} d r+\kappa\right\},
$$

where $a(r)=\int \operatorname{div} X d r$ and $\kappa \in \mathbb{R}$ is a constant of integration.

For linear vector fields there is also a Dulac function in terms of a quadratic function if some spectral condition holds.

Theorem 4 (Linear case [10]). Let $X(z)=A z$ be a linear vector field with $A$ having at most one eigenvalue zero. The linear system (1) does not have periodic orbits if and only if the spectrum of the matrix $A$ consists only of eigenvalues with nonzero real part or zero, i.e., $\sigma(A) \cap i \mathbb{R} \subseteq\{0\}$.

Proof. Let the vector field be $X(z)=A z$ with

$$
\left(\begin{array}{ll}
a & b \\
c & d
\end{array}\right) \in \mathbb{R}^{2 \times 2}
$$

Instead of the general partial differential inequality (2) the author considered

$$
\operatorname{div}(B X)=\|X\|^{2}:=P^{2}+Q^{2}
$$

and made a quadratic ansatz for the Dulac function

$$
B=\frac{1}{2}\langle z, G z\rangle
$$

with some matrix $G \in \mathbb{R}^{2 \times 2}$. Then equation (4) reduces to

$$
A^{T} G+G A+\operatorname{tr} A \cdot G=A^{T} A .
$$

Letting $S:=A+\operatorname{tr} A$, one obtains

$$
S^{T} G+G S=A^{T} A,
$$

Equation (5) is the well-known Lyapunov equation, a special case of the more general Silvester equation. Now one can apply the well-established solvability theory for the Silvester equation [15] and verify that the Lyapunov equation (5) does indeed have a unique solution under the spectral assumptions that were made. On the other hand, one obtains by direct calculation

$$
B=b_{20} x^{2}+b_{02} y^{2}+b_{11} x y+b_{10} x+b_{01} y+b_{00}
$$

values of $b_{i j}$ are shown in Figure 1 . The case $\operatorname{tr} A=$ $a+d=0$ has to be examined with care. The reader may verify that having spectrum $\sigma(A)=\left\{0, \frac{1}{2} \operatorname{tr} A\right\}$ is equivalent to $\operatorname{det} A=\left(\frac{\operatorname{tr} A}{2}\right)^{2}$. Hence the quadratic Dulac function from (6) does the job because we assumed that at most one eigenvalue of $A$ is zero.

The Hartman Grobman theorem combined with the last two results gives some local existence statement holding in some neighborhood of hyperbolic fixed points.

Proposition 1. Let $X$ be a smooth vector field and $z$ a hyperbolic zero of $X$, i.e., the real part $\operatorname{Re} z \neq 0$ is different from zero. Then there is a neighborhood $U$ of $z$ such that $X$ has a Dulac function on $U$.

Remark 2. Proposition 1 says morally that near to hyperbolic equilibriums one can define Dulac functions, which is what we expected since in dimension $n=2$ a hyperbolic equilibrium is either a node (two real eigenvalues of the same sign), a saddle (two real eigenvalues of different sign) or a focus, sometimes also called spiral point, (two complex conjugate eigenvalues with non-zero real part). Can there be a Dulac function near to a non-hyperbolic equilibrium? We believe so, unless the equilibrium is a center. Recall that a non-hyperbolic equilibrium (two purely imaginary eigenvalues of opposite sign) can be either a center or a focus.

\section{Qualitative THEORY}

Let us recall now some basic definitions and results that are frequently used in the qualitative theory of planar differential equations. For a more detailed introduction we refer to [16] and [13]. We call system (1) integrable in domain $\mathcal{D}$ if it has a first integral defined on this domain, i.e., a non-constant smooth scalar-valued function $H$ of class $\mathcal{C}^{k}$ which is constant 
on each solution $(x(t), y(t))$ of (1) as long as it is defined. This means: if $(x(t), y(t))$ is any fixed solution of (1) defined for $t \in\left[0, t_{\max }\right]=: I_{\max }$, its maximal interval of existence, and $H \in \mathcal{C}^{1}(\mathcal{D}, \mathbb{R})$ a first integral of system (1), then there is a real number $h$ such that

$$
H(x(t), y(t))=h \quad \text { for all } \quad t \in I_{\max }
$$

is satisfied. Taking the derivative with respect to time $t$ of equation (7), we see that any first integral $H \in$ $\mathcal{C}^{1}(\mathcal{D}, \mathbb{R})$ of (1) satisfies the linear partial differential equation

$$
\langle\nabla H, X\rangle=P \cdot H_{x}+Q \cdot H_{y}=0 .
$$

Conversely, every non-constant solution of equation (8) is a first integral $H: \mathcal{D} \rightarrow \mathbb{R}$ of (1). If $H_{0}$ is a non-constant solution of (8), then every other solution is of the form $F\left(H_{0}\right)$, where $F$ is an arbitrary function having continuous partial derivatives (use the chain rule to verify this). First integrals are strongly related to the notion of integrating factors.

Definition 2 (Integrating factor). An integrating factor $\mu$ of the planar dynamical system (1) is a smooth solution $\mu \in \mathcal{C}^{1}(\Omega, \mathbb{R})$ of the linear partial differential equation

$$
\operatorname{div}(\mu \cdot X)=\mu \cdot \operatorname{div} X+\langle\nabla \mu, X\rangle=0 \quad \text { in } \Omega .
$$

Note that the first equality in $(9)$ is due to the Leibniz rule and $\operatorname{div} X$, the divergence of the vector field $X$, denotes the trace of the Jacobian of $X, \operatorname{div} X=$ $\frac{\partial P}{\partial x}+\frac{\partial Q}{\partial y}$.

If instead a first integral $H: \mathcal{D} \rightarrow \mathbb{R}$ of $(1)$ is known, using equation (8), one can equivalently define an integrating factor as the common value of the ratios

$$
\mu(x, y)=\frac{H_{y}}{P} \stackrel{!}{=}-\frac{H_{x}}{Q},
$$

i.e., an integrating factor must satisfy both $H_{y}=\mu P$ and $H_{x}=-\mu Q$. The latter two relations can be read as

$$
d H(x, y)=\mu(x, y)(P(x, y) d y-Q(x, y) d x),
$$

where $d H(x, y)$ indicates the differential of $H$. Thus, multiplying the right hand side of (1) by an integrating factor makes the equation an exact differential and an exact equation), which means that, whenever an integrating factor is available, the modified vector field $\mu X$ has vanishing divergence and the problem of solving equation (1) is reduced to one-dimensional integration

$$
H(x, y)=\int_{\left(x_{0}, y_{0}\right)}^{(x, y)} \mu(x, y)(P(x, y) d y-Q(x, y) d x)
$$

Note that the latter line integral might not be welldefined if domain $\mathcal{D}$ is not simply connected. For this reason, integrating factors are usually considered only in connected components of $\mathcal{D}$. Secondly, we observe that the vector fields $X$ and $\mu X$ have the same phase portrait (with maybe reversed orientation if $\mu$ is negative) as long as $\mu$ does not vanish. For this reason, solving system (1), i.e., constructing a first integral and finding an integrating factor for it, are considered to be equivalent problems. In applications the notion of inverse integrating factor is very common.

Definition 3 (Inverse integrating factor). The function $V \in \mathcal{C}^{1}(\Omega, \mathbb{R})$ is called an inverse integrating factor for the planar system (1) in the domain $\Omega \subseteq R^{2}$ if

$$
\mu=\frac{1}{V}
$$

is an integrating factor of system (1) in $\Omega \backslash\{V=0\}$. As usual $\{V=0\}$ is short notation for the preimage of zero under $V, V^{-1}(0)=\{z \in \Omega: V(z)=0\}$.

The method of integrating factors is, both historically and theoretically, a very important technique in the qualitative analysis of first order ordinary differential equations. The use of integrating factors goes back to Leonard Euler (1707-1783).

Integration factors and first integrals refer to the problem of integrating the planar system (1), which means geometrically nothing but finding smooth curves that are tangential to the vector field at each point. Then, another interesting problem is deriving the qualitative behavior of these solution curves as, for instance, their topology (whether they are closed or not), their asymptotics (whether they blow up in finite time or remain in some compact set and approach a limit cycle or an equilibrium point) and stability properties.

\section{A Unifying POINT OF VIEW}

Definition 4 (Invariant curve). Let $\Omega \subseteq \mathbb{R}^{2}$ be an open set of the plane. An invariant curve is the vanishing set or the preimage of zero of some smooth function. More precisely, to a given function $f \in$ $\mathcal{C}^{1}(\Omega, \mathbb{C})$ we associate the preimage of zero

$$
f^{-1}(0) \equiv\{f=0\}:=\{z \in \Omega \mid f(z)=0\}
$$

and call it an invariant curve of the vector field $X=$ $(P, Q) \in \mathcal{C}^{1}\left(\mathcal{D}, \mathbb{R}^{2}\right)$ if there is a smooth function $k \in$ $\mathcal{C}^{1}(\Omega, \mathbb{C})$, called cofactor of $f$, satisfying the relation

$$
\langle\nabla f, X\rangle=k \cdot f \quad \text { for all } z \in \Omega
$$

or more explicitly

$$
f_{x}(z) \cdot P(z)+f_{y}(z) \cdot Q(z)=k(z) \cdot f(z) .
$$

Here $\nabla f=\left(\frac{\partial f}{\partial x}, \frac{\partial f}{\partial y}\right)^{T}$ denotes the gradient of $f,\langle\cdot, \cdot\rangle$ is the canonical inner product of $\mathbb{R}^{2}$ and the subscripts of $f$ indicate partial derivates, $f_{x}=\frac{\partial f}{\partial x}, f_{y}=\frac{\partial f}{\partial y}$. Note that on the invariant curve $\{f=0\}$ the gradient of $f$, $\nabla f$, is orthogonal to the vector field $X$ by the defining 
property of invariant curves 13 . By convention, the function $f$ defining the invariant curve $f^{-1}(0) \subseteq \mathbb{R}^{2}$ is called the invariant function.

The notion of exponential factors, a special case of invariant functions, is useful for studying the multiplicity of invariant curves. It allows the construction of first integrals for polynomial systems via the same method used by Darboux.

Definition 5 (Exponential factor). Given two $h, g \in \mathbb{R}[x, y]$ coprime polynomials, the function $\exp (g / h)$ is called an exponential factor for system (1) if there is a polynomial $k \in \mathbb{R}[x, y]$ of degree at most $d-1, d:=\max \{\operatorname{deg} P, \operatorname{deg} Q\}$ being the degree of the polynomial system (1), satisfying the relation

$$
\left\langle\nabla\left(\mathrm{e}^{g / h}\right), X\right\rangle=k \cdot \mathrm{e}^{g / h} .
$$

Note that obviously $\left\{\left(\mathrm{e}^{g / h}\right)=0\right\}=\emptyset$, but $\{g=0\}$ defines an invariant curve for system (1).

Definition 6 (Darboux function). Any function of the form

$$
\prod_{i=1}^{r} f_{i}^{\lambda_{i}} \prod_{j=1}^{l}\left(\exp \left\{g_{j} / h_{j}^{n_{j}}\right\}\right)^{\mu_{j}}
$$

where, for $1 \leq i \leq r$ and $1 \leq j \leq l, f_{i}(z)=0$ and $g_{j}(z)=0$ are invariant curves for system (1), $h_{j}$ is a polynomial of $\mathbb{C}[x, y], \lambda_{i}$ and $\mu_{j}$ are complex numbers and $n_{j}$ is a natural number or zero, is called Darboux function.

The following remark reminds the reader of some facts about invariant curves.

Remark 3. (1.) Invariant curves are very important in the qualitative study of dynamical systems because they generalize the notion of integrating factors and Dulac functions. Thus, it is possible to interpret integrating factors and Dulac functions as invariant functions to certain cofactors: an integrating factor is nothing but an invariant function having cofactor

$$
k=-\operatorname{div} X
$$

and a Dulac function is an invariant function with cofactor

$$
k=-\operatorname{div} X+\frac{1}{f} \cdot g,
$$

$g$ being a continuous function with $g>0$ for almost every $z \in \Omega$. Note that the latter interpretation of a Dulac function as an invariant function to a specific cofactor makes sense only when $f$ does not vanish, i.e., the Dulac function is not defined respectively singular on the invariant curve, the vanishing set of the invariant function. This already gives a clue to the natural boundaries on the maximal domain of definition of Dulac functions.
(2.) An easy observation is that, if $f$ and $g$ are invariant functions with cofactor $k_{f}$ and $k_{g}$, respectively, then their pointwise product $f \cdot g$ also defines an invariant curve with cofactor $k_{f}+k_{g}$.

(3.) Without loss of generality, we will always consider complex-valued invariant functions because, if $f$ is an invariant function with cofactor $k$ (with respect to some vector field in some domain), then its conjugate function $\bar{f}$ is also an invariant function having cofactor $\bar{k}$, and therefore the product $f \cdot \bar{f}$ is a real-valued invariant function with cofactor $k+\bar{k}$. The same holds for exponential factors.

(4.) In the case of polynomial planar vector fields, the algebraic part of invariant curves and exponential factors has already been developed. We quote some of these results and refer to [17], 18] and [19] for further reading. Therefore, let the vector field $X \in \mathbb{R}_{d}^{2}[x, y]$ be a polynomial vector field of degree $d$. Furthermore, assume that $\frac{d(d+1)}{2}+1$ different irreducible invariant algebraic curves are known. Then one can construct a first integral of the form

$$
H=f_{1}^{\lambda_{1}} \cdot \ldots \cdot f_{s}^{\lambda_{s}}
$$

where each $f_{i}(x, y)$ defines an invariant algebraic curve

$$
f_{i}(x, y)=0
$$

for system (1) and $\lambda_{i} \in \mathbb{C}$, not all of them null, for $i=1,2, \ldots, s, s \in \mathbb{N}$. The functions of type 15 are called Darboux functions.

(5.) The irreducibility of the invariant functions in the algebraic case must be replaced by the condition

$$
\begin{aligned}
\{p \in \Omega: f(p)=0 \text { and } & \nabla \\
& f(p)=0\} \\
& \subseteq\{p \in \Omega: X(p)=0\}
\end{aligned}
$$

in the non-algebraic case.

(6.) An easy observation: Any invariant curve $\{f=$ $0\}$ has exactly one cofactor

$$
k=\frac{\langle\nabla f, X\rangle}{f} .
$$

\section{Open questions}

In Section 2, Proposition 1, we obtained a local existence result for Dulac functions near to a hyperbolic equilibrium. The proof was based on theorem 4 , where we calculated a Dulac function explicitly in terms of a quadratic polynomial. In this proof we observed, in fact, why one needs to impose that at most one eigenvalue of matrix $A$ is zero, because, if we had two such eigenvalues $\sigma(A)=\{0\}$, the matrix would have trace zero and we would have divided by it. Then, by applying the Hartman Grobman theorem, the result carried over to hyperbolic fixed points. Observe that neither does the Hartman Grobman theorem hold for equilibria $p$ with linearization having a purely imaginary or zero spectrum, nor could we make any use 


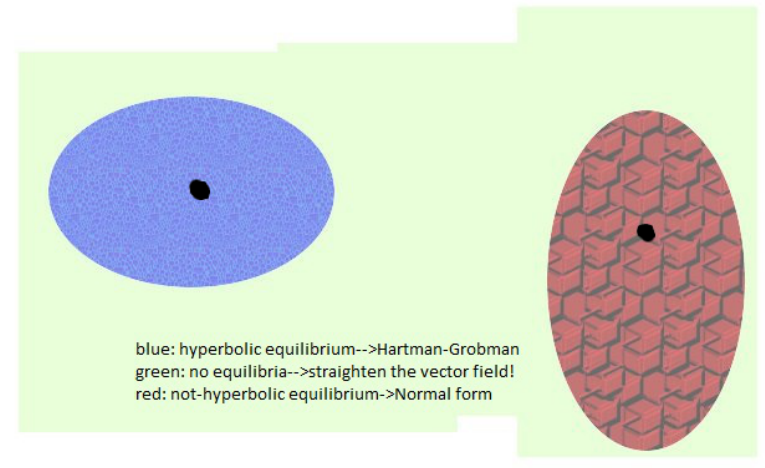

Figure 2. Gluing three different diffeomorphisms

of it, because in both cases $\operatorname{div} X(p)=0$ holds and our Dulac function blows up. Hence, in future the following question for nonlinear vector fields has to be addressed:

Question 2. When does a Dulac function exist near to non-hyperbolic fixed points?

This question is somehow naive, because one has to deal here with the center-focus-problem, which is still not completely solved in general. However, this Dulac approach may give a new perspective on it. Why do we bother about all these local existence statements for Dulac functions? In principle, the motivation arose from the following

\section{Algorithm 1.}

Input: a nonlinear planar vector field $X$;

Output: number and position of periodic orbits together with phase portrait.

Step1: Determine the zeros of $X$.

Step2: At each zero, define locally a Dulac function.

Step3: Extend them as long as possible. If no further extension is possible, one has found the limit cycles and determined the phase portrait.

Of course, this is rather a pseudo algorithm, because one cannot accomplish any of its steps. One more approachable but somehow technical step is to combine these local results in a global one. Technically, this means, as one has to deal here with different local coordinate representations, that one has to glue together different diffeomorphisms. Summing up, we claim that the Dulac method in the spirit of algorithm 1 will give new insights in the qualitative theory of planar differential equations.

\section{REFERENCES}

[1] I. Burdujan. Some geometrical aspects of the theory of quadratic differential equations. Bul Inst Politehn Iaşi Secţ I 37(41)(1-4):39-44, 1991.
[2] D. K. Kayumov. Limit cycles of a class of quadratic differential equations. In Problems in the theory of ordinary differential equations (Russian), pp. 25-29, 109-110. Samarkand. Gos. Univ., Samarkand, 1984.

[3] D. E. Koditschek, et al. Limit cycles of planar quadratic differential equations. J Differential Equations 54(2):181-195, 1984.

[4] Y. S. Il'yashenko. Finiteness theorems for limit cycles, vol. 94 of Translations of Mathematical Monographs. American Mathematical Society, Providence, RI, 1991. Translated from the Russian by H. H. McFaden.

[5] H. Dulac. Sur les cycles limites. Bull Soc Math France 51:45-188, 1923.

[6] J. Écalle, et al. Non-accumulation des cycles-limites. I. C R Acad Sci Paris Sér I Math 304(13):375-377, 1987.

[7] Y. Ilyashenko. Centennial history of Hilbert's 16th problem. Bull Amer Math Soc (NS) 39(3):301-354, 2002.

[8] Y. S. Il'yashenko. Centennial history of Hilbert's 16th problem. In Fundamental mathematics today (Russian), pp. 135-213. Nezavis. Mosk. Univ., Moscow, 2003.

[9] J. Llibre. On the 16-Hilbert problem. Gac R Soc Mat Esp Preprint 2012.

[10] M. Himmel. On the existence of periodic orbits of ordinary differential equations (transl.) pp. 43-46, 2009.

[11] A. A. Andronov, et al. Qualitative theory of second-order dynamic systems. Halsted Press (A division of John Wiley \& Sons), New York-Toronto, Ont., 1973. Translated from the Russian by D. Louvish.

[12] H. P. Guckenheimer, John. Nonlinear oscillations, dynamical systems, and bifurcations of vector fields. Springer-Verlag.

[13] F. Dumortier, et al. Qualitative theory of planar differential systems. Universitext. Springer-Verlag, Berlin, 2006.

[14] D. A. Neumann. Classification of continuous flows on 2-manifolds. Proc Amer Math Soc 48:73-81, 1975.

[15] R. Bhatia, et al. How and why to solve the operator equation $a x-x b=y \mathbf{2 9 : 1 - 2 1 , 1 9 9 7 . ~}$

[16] I. A. García, et al. A survey on the inverse integrating factor. Qual Theory Dyn Syst 9(1-2):115-166, 2010.

[17] J. Llibre. Integrability of polynomial differential systems. In Handbook of differential equations, pp. 437-532. Elsevier/North-Holland, Amsterdam, 2004.

[18] D. Schlomiuk. Algebraic particular integrals, integrability and the problem of the center. Trans Amer Math Soc 338(2):799-841, 1993.

[19] D. Schlomiuk. Algebraic and geometric aspects of the theory of polynomial vector fields. In Bifurcations and periodic orbits of vector fields (Montreal, PQ, 1992), vol. 408 of NATO Adv. Sci. Inst. Ser. C Math. Phys. Sci., pp. 429-467. Kluwer Acad. Publ., Dordrecht, 1993. 